\title{
ARTICLE OPEN Deciphering the regulatory and catalytic mechanisms of an unusual SAM-dependent enzyme
}

Qiu Sun (iD), Yuehong $\mathrm{Hu}^{1}$, Yijun $\mathrm{Gu}^{2}$, Jiangkun Huang ${ }^{3}$, Jun $\mathrm{He}^{1}$, Lan Luo ${ }^{3}$, Yi Yang ${ }^{4}$, Shuo Yin ${ }^{4}$, Chao Dou ${ }^{1}$, Tianqi Wang ${ }^{1}$, Xianghui Fu ${ }^{1}$ Ling $\mathrm{He}^{3}$, Shiqian $\mathrm{Qi}^{1}$, Xiaofeng $\mathrm{Zhu}^{1}$, Shengyong Yang ${ }^{1}$, Xiawei Wei ${ }^{1}$ and Wei Cheng ${ }^{1}$

S-adenosyl-1-methionine (SAM)-dependent enzymes regulate various disease-related behaviors in all organisms. Recently, the leporin biosynthesis enzyme Lepl, a SAM-dependent enzyme, was reported to catalyze pericyclic reactions in leporin biosynthesis; however, the mechanisms underlying Lepl activation and catalysis remain unclear. This study aimed to investigate the molecular mechanisms of Lepl. Here, we reported crystal structures of Lepl bound to SAM/5'-deoxy-5'-(methylthio) adenosine (MTA), Sadenosyl-homocysteine (SAH), and SAM/substrate states. Structural and biochemical analysis revealed that MTA or SAH inhibited the enzyme activities, whereas SAM activated the enzyme. The analysis of the substrate-bound structure of Lepl demonstrated that this enzymatic retro-Claisen rearrangement was primarily driven by three critical polar residues His133, Arg197, Arg295 around the active site and assisted by SAM with unclear mechanism. The present studies indicate that the unique mechanisms underlying regulatory and catalysis of the unusual SAM-dependent enzyme Lepl, not only strengthening current understanding of the fundamentally biochemical catalysis, but also providing novel insights into the design of SAM-dependent enzyme-specific small molecules.

Signal Transduction and Targeted Therapy (2019)4:17; https://doi.org/10.1038/s41392-019-0052-y

\section{INTRODUCTION}

Antibiotic resistance is a major concern worldwide, and new therapeutic drugs are urgently needed. ${ }^{1-3}$ Fungi are an invaluable resource of antibiotic candidates. ${ }^{4-7}$ Pyridone alkaloids (e.g., PF1140, leporins, and fusaricides) are present in numerous fungi, displaying diverse biological activities and applied for various pharmaceutical purposes. ${ }^{8,9}$ Owing to the complexity of pyridone alkaloids with a chiral center and pure forms, the efficient synthesis of these compounds has drawn increasing attention from chemists and biochemists. ${ }^{10-14}$ Pericyclic reactions are facile access to obtain various biomolecules including some pyridone alkaloids and have been considered as a key transformational process in the biosynthesis of many natural compounds. ${ }^{15-18}$ However, enzymes catalyzing pericyclic reactions rarely exist naturally. ${ }^{19-21}$ Recently, the novel multifunctional S-adenosyl-1methionine (SAM)-dependent enzyme Lepl, located in the PKSNRPS gene cluster for leporin biosynthesis, was characterized in Aspergillus flavus. ${ }^{22,23}$ Ohashi et al. first reported this multifunctional enzyme in catalyzing pericyclic reactions for leporin biosynthesis. ${ }^{22}$ Lepl is considered as a SAM-dependent methyltransferase that can catalyze stereoselective dehydration via three pericyclic transformational processes: intramolecular Diels-Alder and hetero-Diels-Alder reactions and a retro-Claisen rearrangement. ${ }^{22,24}$
The detailed mechanisms of SAM-dependent enzymatic actions are well understood, ${ }^{25,26}$ whereas the mechanism underlying Leplcatalyzed pericyclic reactions remains unclear. ${ }^{22,24}$

This study aimed to investigate the mechanism of Lepl action. Herein, a series of structures, including those of SAM $/ 5^{\prime}-$ deoxy- $5^{\prime}$ (methylthio) adenosine (MTA)-bound Lepl, S-adenosyl-homocysteine (SAH)-bound Lepl, and SAM/substrate-bound Lepl, and various biochemical analyses helped elucidate the mechanisms underlying Lepl activation and catalysis. The present study not only elucidates the novel mechanism of SAM-dependent enzymatic action but also provides potential insight regarding similar enzymes involved in various physiological processes. The findings of this study will facilitate the development of new enzymes for applications in chemistry and synthetic biology.

\section{RESULTS}

Similar chemicals regulate Lepl activity

Although pericyclic reactions are indispensable for the biosynthesis of many natural compounds, ${ }^{20}$ enzyme-catalyzed pericyclic reactions are rare in nature. Lepl, an unusual SAM-dependent enzyme, can catalyze pericyclic transformations involved in the retro-Claisen rearrangement ${ }^{22}$ (Supplemental Fig. 1). However, the

\footnotetext{
${ }^{1}$ Division of Respiratory and Critical Care Medicine, Center of Infectious Diseases, National Clinical Research Center for Geriatrics and State Key Laboratory of Biotherapy, West China Hospital of Sichuan University and Collaborative Innovation Center of Biotherapy, Chengdu 610041, China; ${ }^{2}$ Shanghai Synchrotron Radiation Facility, Zhangjiang Lab, Zhangheng Road 239, Pudong District, Shanghai 201203, China; ${ }^{3}$ Department of Medicinal Chemistry, Key Laboratory of Drug-Targeting and Drug Delivery System of the

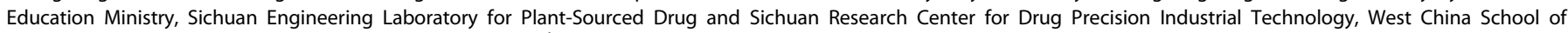
Pharmacy, Sichuan University, Chengdu 610041, China and ${ }^{4}$ West China School of Public Health, Sichuan University, Chengdu 610041, China

Correspondence: Xiawei Wei (xiaweiwei@scu.edu.cn) or Wei Cheng (chengwei669@scu.edu.cn)

These authors contributed equally: Qiu Sun, Yuehong Hu, Yijun Gu
}

Received: 22 April 2019 Revised: 28 April 2019 Accepted: 7 May 2019

Published online: 24 May 2019 


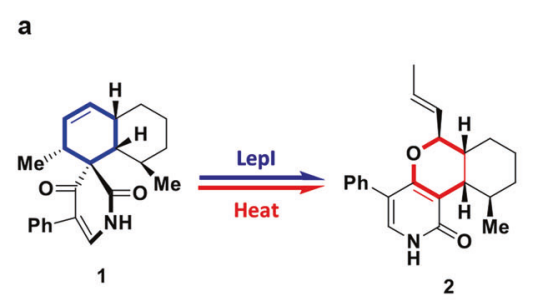

2
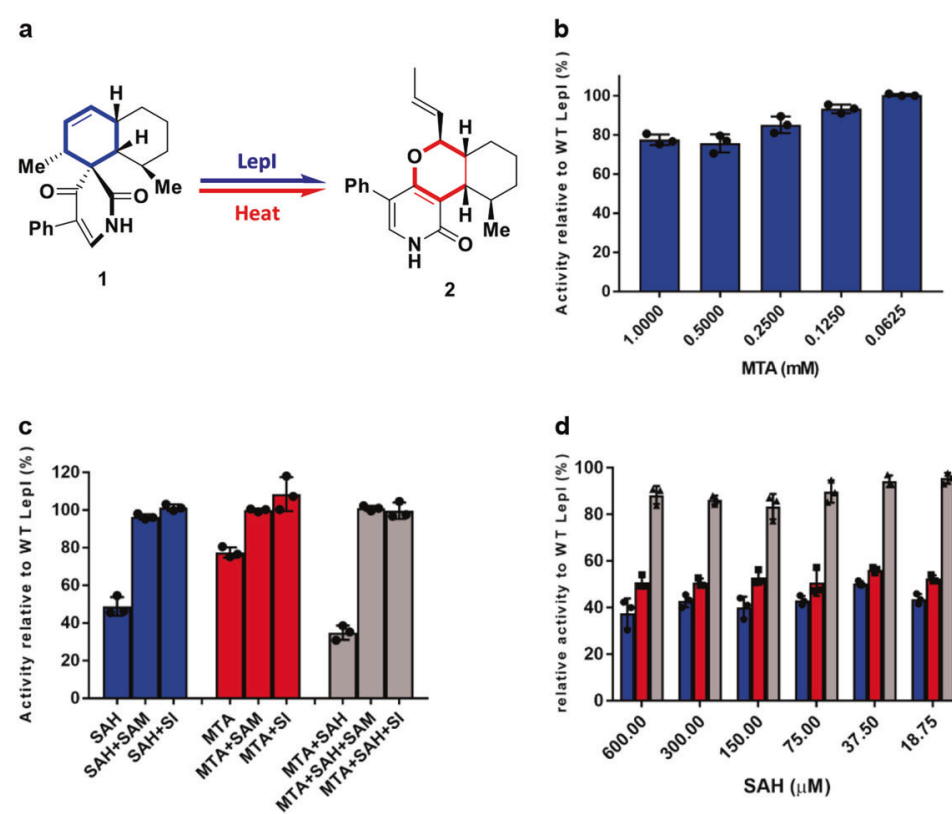

d

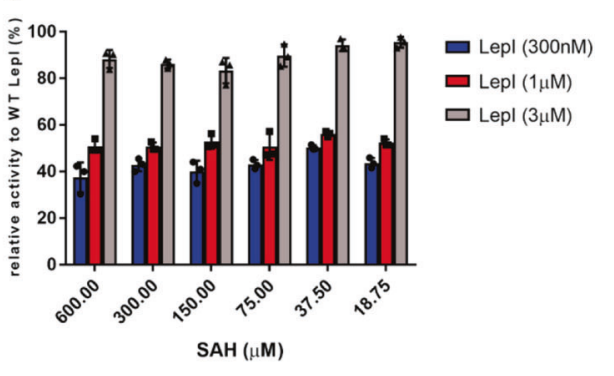

Fig. 1 Similar chemicals regulate Lepl activity. a Scheme for Lepl-catalyzed retro-Claisen rearrangement. The structures show the relative stereochemistry. $\mathbf{b}$ Inhibition of MTA on Lepl-catalyzed retro-Claisen rearrangement under different concentrations in the presence of $300 \mathrm{~nm}$ Lepl. c Recovery of retro-Claisen rearrangement by $1 \mathrm{~mm}$ SAM or SI in the presence of $1 \mathrm{~mm}$ SAH or $1 \mathrm{~mm}$ MTA and $300 \mathrm{nM}$ Lepl. The experiments in b-d were performed three times, each with three biological replicates. Data are the mean \pm S.D. d Inhibition of SAH on Leplcatalyzed retro-Claisen rearrangement under different concentrations in the presence of $0.3,1.0$, or $3.0 \mu \mathrm{m}$ Lepl

mechanisms underlying Lepl activation and catalysis remain unclear, although Lepl shares considerable sequence identity within the SAM enzyme superfamily (Supplemental Fig. 2).

In this study, we performed an enzymatic assay using Lepl to catalyze compound 1 to leporin C (Fig. 1a, Supplemental Fig. 1). Because some endogenously bound MTA and SAM copurified with Lepl (Supplemental Fig. 3), we examined Lepl enzymatic activity in the presence of MTA and simultaneously assessed Lepl activity using the competitive inhibitor $\mathrm{SAH}^{27}$ MTA moderately decelerated the retro-Claisen rearrangement of Lepl when converting compound $\mathbf{1}$ to leporin $\mathrm{C}$, decreasing its activity by $30 \%$ at $0.5 \mathrm{~mm}$ (Fig. 1b). Notably, MTA inhibited Lepl activity in a concentration-dependent manner (Fig. 1b), whereas SAH decreased Lepl activity by $50 \%$ in a dose-independent manner, slightly altered at enzyme concentrations of $0.3 \mu \mathrm{m}$ and $1.0 \mu \mathrm{m}$, and even those approaching 3.0 $\mu \mathrm{m}$ (Fig. 1d). Remarkably, no inhibition was observed at a Lepl concentration of $\sim 3 \mu \mathrm{m}$. Intriguingly, the synergistic effect of MTA in the presence of SAH decreased Lepl activity by $\sim 70 \%$ (Fig. 1c). Furthermore, we investigated whether the positively charged SAM and the corresponding analog Sinefungin (SI) could rescue Lepl activity when the enzyme was treated with MTA, SAH or both. Consistent with previous report, ${ }^{22}$ both SAM and SI completely rescued Lepl activity for retro-Claisen rearrangement (Fig. 1c). This result prompted the following questions: why do MTA and SAH have synergistic effects? What are the different mechanisms underlying MTA and SAH inhibition? How can SAM/SI rescue Lepl activity? We postulated that the mechanisms underlying activation or inhibition by these compounds could be distinct from those of other members of the SAM-dependent enzyme superfamily; this postulation was supported by structural and biochemical findings.

Structures of the Lepl complex

To elucidate the mechanism of Lepl action, we first determined the structure of SAM/MTA-bound Lepl at a high resolution of $1.7 \AA$ (Fig. 2a, b, Extended Data Table 1). The overall structure was found to be largely similar to that of SAM-dependent enzymes (Supplemental Fig. $4 a-d),{ }^{28-31}$ exhibiting a core $a / \beta$ fold
(Supplemental Fig. 4e) of alternating $\beta$ strands $(\beta 1-\beta 7)$ and $\alpha$ helices (a1-a19) (Fig. 2a). This structure exhibiting $a$ helices and $\beta$ sheets comprises an all-helix amino-terminal domain (NTD), a carboxy-terminal domain including a substrate-binding site, and a SAM-binding site (Fig. 2b, Supplemental Fig. 5), which is formed by a seven-stranded $\beta$ sheet rounded with five helices. Two molecules of Lepl observed in an asymmetric crystal unit form the homodimer, which is primarily mediated by the NTD (Supplemental Fig. 5a); specifically, the a1 and a2 segments of one subunit interact with those of another subunit to form interlocking fingers (Supplemental Fig. 5b), and $a 3$ is involved in the formation of the hind surface of the active site (Supplemental Fig. 5c). To verify the importance of $a 1$ and $a 2$ in the dimers for enzymatic activity, we performed an enzymatic assay with a1 single deletion or a1-a2 double deletion of Lepl. These deletions, particularly a1a2 double deletion, completely obliterated the enzymatic activity (Fig. 2d, e, Supplemental Fig. 5d, e). Moreover, the a1 single deleted enzyme displayed stable variable conformations in a sizeexclusion chromatographic assay. We then comprised enzymatic assays using each corresponding fraction (Fig. 2d, e). These results indicate that the $a 1$ and $a 2$ segments play an important role in Lepl homo-oligomerization, which is essential for its activity.

As determined from the strong electron density, SAM binds to one side of the Lepl pocket (Fig. 2b, Supplemental Fig. 6a, b) and maintains the catalytic domain in the active state through interaction networks. The adenosine rings and amino acids of SAM interact with Phe276 and Asn275 via $\pi-\pi$ interaction and salt bonding, respectively. $\mathrm{O} 4$ of the ribose and the tail amide of SAM form a pair of hydrogen bonds with the main chain of Gly227. In addition, Arg291 forms a pair of hydrogen bonds with the $O$ atom and amino group of the SAM tail via the side chain and main chain, respectively. Specifically, a hydrogen bond forms between the $\mathrm{S}^{+}-\mathrm{CH}_{3}$ moiety and the main chain of Leu292. In addition, lle293 also forms a hydrogen bond with C5 of SAM. Collectively, these interactions result in the coordination of SAM in an extended confirmation (Fig. 2c).

Additional electron density was observed in the pocket of the Lepl density map, located opposite the SAM-binding site. As MTA 
a

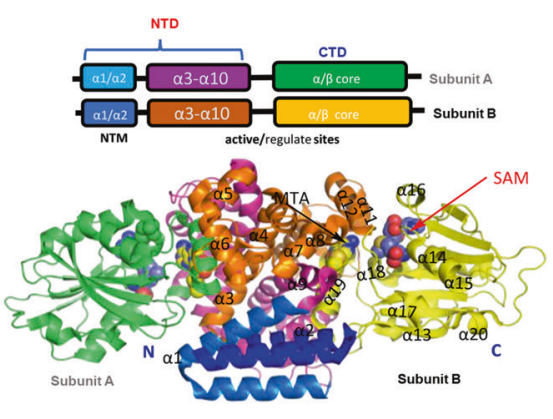

b

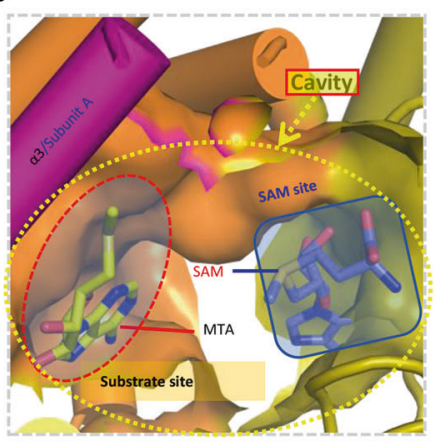

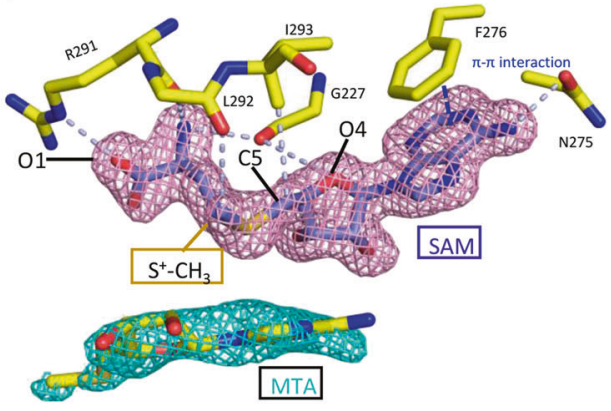

d

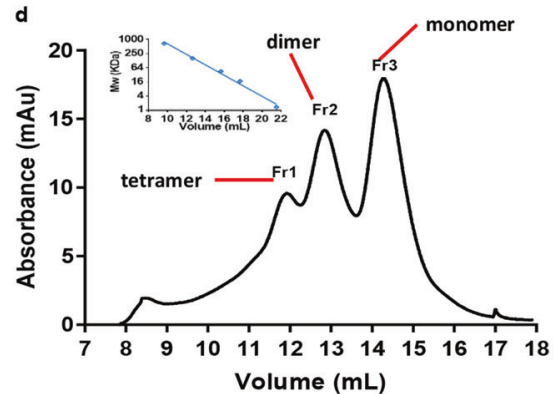

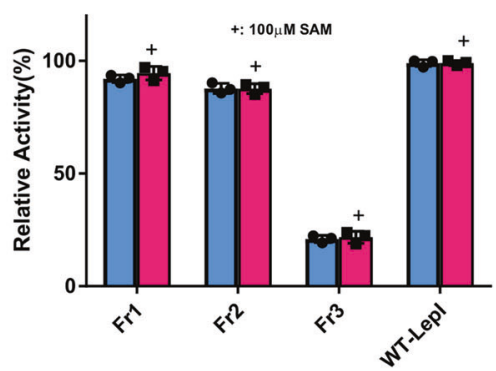

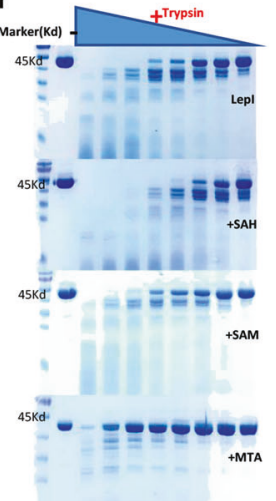

Fig. 2 Structure of Lepl in complex with MTA and SAM. a Architecture of the Lepl dimers in complex with SAM and MTA. Lepl adopts a SAMdependent MT fold. Small molecules are indicated as spheres in the cavity of Lepl structure. $\mathbf{b}$ The cavity comprises two sites: a SAM site and a substrate site. The close-up stereo view of the mimic substrate MTA and SAM-binding site indicates that the SAM (indicated by the red circle) site is independent of the substrate site (indicated by the green rectangle). c The 2Fo-Fc electron densities for MTA (colored cyan) and SAM (colored pink) at $1 \sigma$ and $2 \sigma$, respectively. A close-up view of the detailed interaction between SAM and Lepl is shown; gray dashed lines indicate the hydrogen bonds, and the blue dashed line indicates the $\pi-\pi$ interaction. $\mathbf{d}$ Analytic gel-filtration of purified Lepl- $\Delta 15$. Three peaks appear, representing the formation of Lepl monomer, dimer, and tetramer according to the standard protein marker. A representative image from three replicate experiments is shown. e Enzymatic activity of Lepl- $\Delta 15 \mathrm{Fr} 1-\mathrm{Fr} 3$ compared with wild-type (WT) Lepl determined through retro-rearrangement assay with triplicate measurement. (Data represent the mean \pm s.d.) The Fr3 monomer indicated in the gel-filtration assay is almost inactive, whereas both Fr1 and Fr2 still have full activity. $\mathbf{f}$ Limited proteolysis of Lepl in the presence of SAM, SAH, or MTA at gradient concentrations of trypsin. The proteolytic fragments were detected by SDS-PAGE and Coomassie staining

could be detected via HPLC analysis of the purified proteins compared with the reference standard of SAM and MTA (Supplemental Fig. 3a, b), we modeled MTA in the electrondense cluster and found it likely to be coordinated in the hydrophobic site (Supplemental Fig. 6c), with some residues displaying significant conformational changes upon MTA binding (Fig. 2e, Supplemental Fig. 6d). Consistent with the structural observations, limited proteolysis assays indicated that purified Lepl treated with MTA exhibited increased trypsin resistance, whereas SAH seemingly decreased trypsin resistance (Fig. 2f). We postulated that the MTA-binding site is the primary channel for substrates or products. The co-factor SAM is too remote from the substrate-binding site; thus, we speculated the electrostatic influence of the SAM sulfonium moiety for catalysis is limited.

To better understand the mechanism underlying Lepl inhibition by $\mathrm{SAH}$, we determined the structure of SAH-bound Lepl at a $2.7 \AA$ resolution (Fig. $3 a, b$ and Extended Data Table 1). The overall architectures of SAM/MTA-bound Lepl and SAH-bound Lepl seemed similar (Fig. 3c). SAM or SAH binding with the enzyme occurs at the inner surface of the pocket and is primarily mediated via ionic bonds, hydrogen bonds and $\pi-\pi$ interactions (Fig. 3d). No hydrogen bonds form between Leu292 or lle293 and SAH because of the absence of a methyl group, which decreases the binding affinity between the region (amino-acid residues 291-296) and $\mathrm{SAH}$. However, based on structural observation, one additional salt bond appears to form between Arg291 and SAH. The close similarity between these two complexes revealed that the reactivity of Lepl might result from the hydrogen bonding and positively charged nature of SAM vs SAH, but not related to structural reason.

Lepl activation and inhibition

Structural analysis in the present study revealed that SAM or SAH occupied a site on the inner surface of the pocket of Lepl that was distinct from the MTA substrate-binding area (Figs. 2b, 3e, Supplemental Fig. 6a). Thermal unfolding assays revealed that SAM, SAH, and MTA increased the melting temperature $(T \mathrm{~m})$ of Lepl by $\sim 6.2^{\circ} \mathrm{C}, 4.3^{\circ} \mathrm{C}$, and $4.4^{\circ} \mathrm{C}$, respectively (Supplemental Fig. $7 \mathrm{a}, \mathrm{b})$. Consistent with the structural observations and thermal assays, limited proteolysis assays indicated increased trypsin resistance in purified Lepl in the presence of SAM or MTA, whereas SAH had negligible effects (Fig. 2f). Furthermore, MTA increased trypsin resistance to a greater extent than did SAM, suggesting that SAM can most likely stabilize Lepl for activation, whereas MTA mimicked the substrate in the active site.

To validate the SAM-binding sites, we individually mutated the residues involved in SAM-binding via enzymatic assays. Concurrent with the results from structural analyses, Gly227Ala (G227A) substitution greatly decreased Lepl activity, whereas Phe276 and Leu292 substitutions showed only a small loss in activity compared with wild-type Lepl, and no activity was detected from Asn275Ala, Arg291Ala, and Ile293Ala, as no protein was obtained 


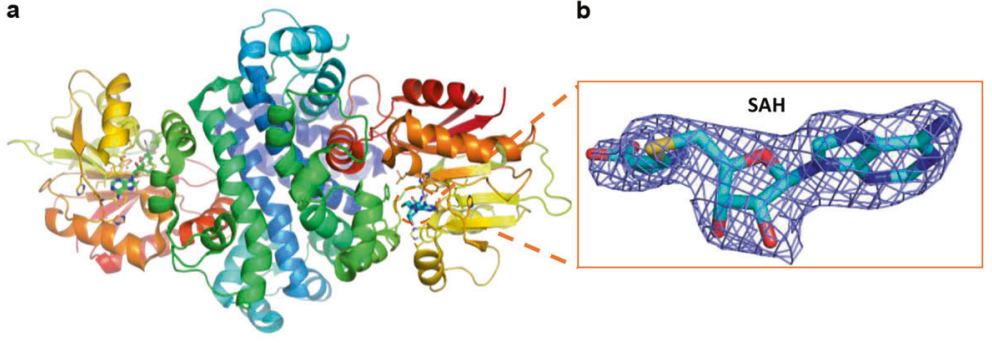

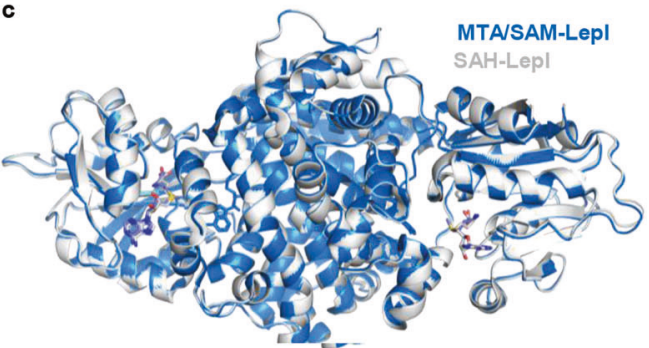

d

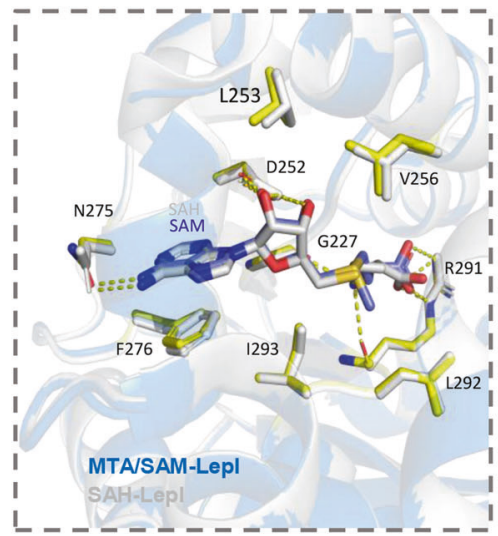

e

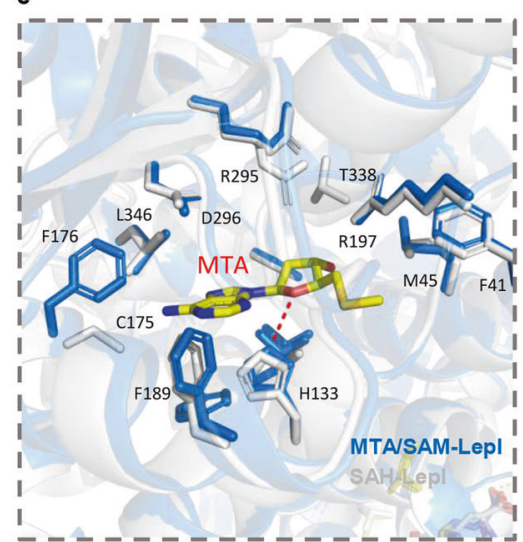

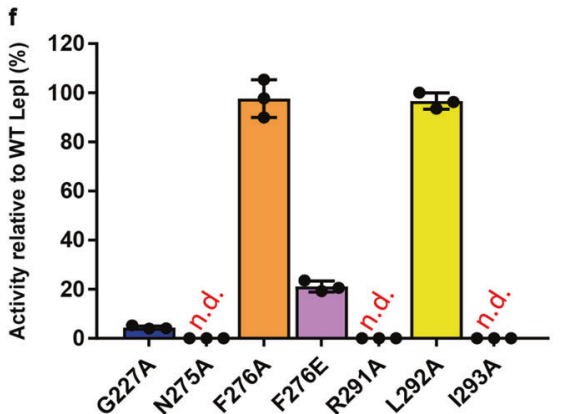

Fig. 3 Activity regulation by SAH/SAM. a Schematic of the overall structure of Lepl in complex with SAH. b The density map of SAH. The 2 Fo-Fc omit map, contoured at 1.5 $\sigma$. The SAH molecule is shown in stick. c Structural comparison of MTA/SAM-Lepl (blue) and SAH-Lepl (gray). d Close-up view of the SAM site between structures of MTA/SAM-Lepl (blue) and SAH-Lepl (gray). Interactions between SAM and residues (G227, N275, and F276) of Lepl are indicated by yellow dashed lines. e Close-up view of the substrate site from MTA/SAM-Lepl (blue) and SAHLepl (gray) structures based on the overall structural alignment. MTA is located at the hydrophobic channel. $\mathbf{f}$ Enzymatic assays of the key residues around the SAM-binding site with variants, n.d. represents no detection of activity, (data represent the mean \pm s.d.)

(Fig. 3f). These substitutions confirmed the importance of residues at the SAM-binding site, as revealed via the complex structure.

As shown in Fig. 1, MTA-and SAH-decelerated Lepl activity; however, the underlying mechanisms remain unclear. MTA probably mimics the substrate binding at the active site, thereby blocking the entry of a native substrate as a competitive inhibitor (Fig. 1b), thereby decreasing Lepl activity. Although SAH-mediated Lepl inhibition probably proceeds via the failure of a stable interaction between SAH and Lepl. Applying SAH and MTA simultaneously almost obliterated Lepl activity, indicating that $\mathrm{SAH}$ and MTA have synergistic effects with different underlying inhibitory mechanisms (Fig. 1c).

Overall, the present results suggest that SAM may stabilize one active state among many available Lepl conformations through substrate mimicry, thereby activating the pericyclic reaction; however, SAH cannot completely activate the pericyclic reaction of Lepl because it lacks of sulfonium moiety for catalysis, whereas MTA occupies the substrate site.

Mechanism underlying Lepl catalysis via the pericyclic reaction To further explore the catalytic mechanism of Lepl, we determined the structure of the Lepl-substrate complex at $2.2 \AA$ (Fig. $4 a$, b, Extended Data Table. 1). The compound 1 was present at the substrate site, which is a hydrophobic pocket with a semiopen configuration (Fig. 4c, Supplemental Fig. 8). Three positive residues (His133, Arg197, and Arg295) and one negative residue (Asp296) are present along with some bulky hydrophobic residues (Phe41, Phe165, Phe169, Phe176, Trp178, and Phe189) around the compound, coordinated in a curve confirmation via salt bonds, hydrogen bonds, and $\pi-\pi$ interactions (Fig. 4c). The polar residue Arg197 is proximal to the C3-C4 double bond, and this positively charged residue has been proposed to decrease C4 electron density of the olefin in favor of potential attack by the $2^{\prime}$-carbonyl oxygen. Arg295 forms hydrogen bonds with an oxygen atom ( $\left(1^{\prime} \mathrm{O}\right)$ of the carbonyl group of compound $\mathbf{1}$ and may be involved in providing a positively charged electrostatic environment for the reaction; moreover, Asp296 also interacts with the compound 1 via hydrogen bonding (Fig. 4c). Notably, His133 not only forms an instant hydrogen bond with 2'-carbonyl oxygen but also probably forms a $\pi$-stacking interaction with the imidazole ring (Fig. 4c). Simultaneously, the active site is formed with the assistance of the bulky hydrophobic residues including Phe41, Phe165, Phe169, Phe176, Trp178, and Phe189, which form $\pi-\pi$ and hydrophobic interactions to maintain a hydrophobic environment. Therefore, to induce the retro-Claisen rearrangement reaction, His133, Arg295, and Asp296 participate in coordinating the substrate in the chair conformation with the assistance of other residues; thereafter, together with Arg197, they provide electrostatic charges to catalyze the rearrangement (Fig. 4c).

To verify important residues around the substrate, we performed an enzymatic assay using a panel of Lepl variants harboring amino-acid substitutions within the substrate-binding site (Fig. 4d, e). Consistent with structural observations, replacing the substrate-interacting residues with alanine significantly reduced catalytic activity. Specifically, mutating His133, Arg197, and Arg295 to alanine, and Asp296 to glutamic acid impaired enzymatic activity (Fig. 4d). Similar results were obtained upon mutation of Ile342 to serine (I342S; Fig. 4e), where this mutation potentially converted a hydrophobic residue to a polar residue to thereby alter the catalytic environment. Notably, mutating the bulky residue Phe41 (F41Y), which packs against the phenolic moiety of compound 1, retained appreciable catalytic activity. We individually replaced other bulky hydrophobic residues (Phe165, Phe169, Phe176, Trp178, Phe189, and Phe297) adjacent to the substrate with alanine or tyrosine. We could not obtain the protein of these variants (Phe41Ala, Phe169Ala, Phe176Ala, and Phe297Ala). These results suggested that these residues formed a hydrophobic site and prevented other molecules, including 
a

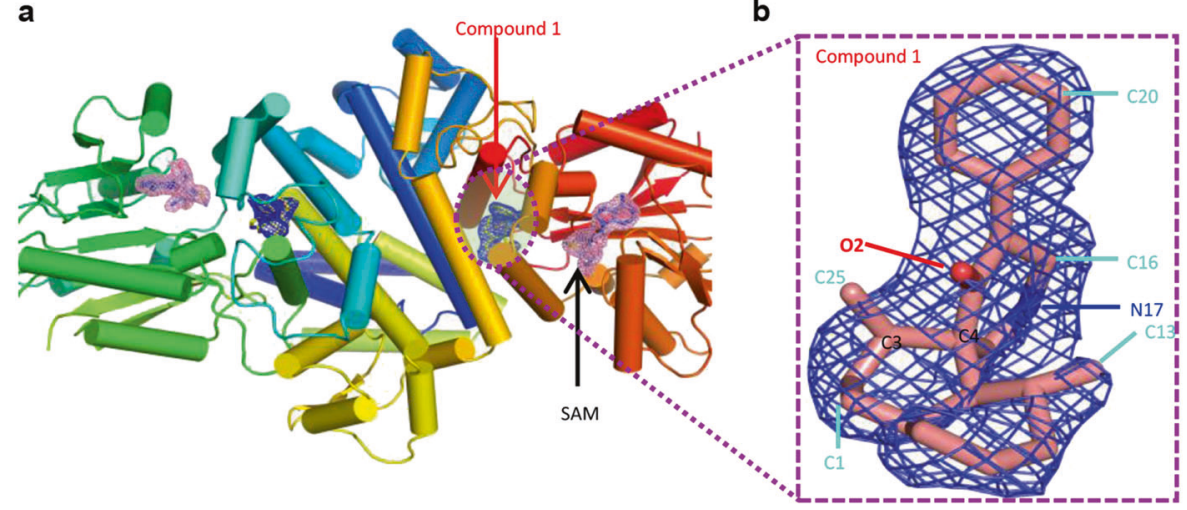

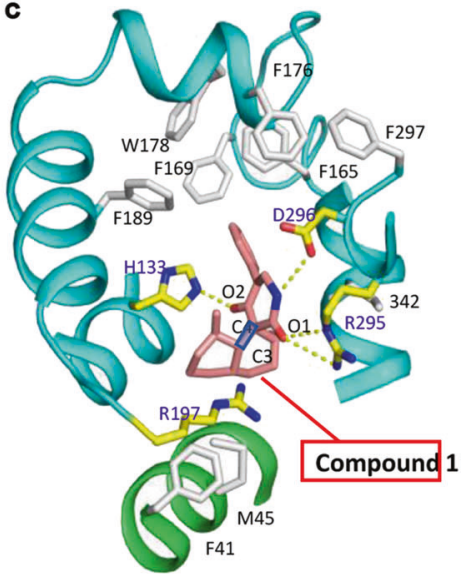

d

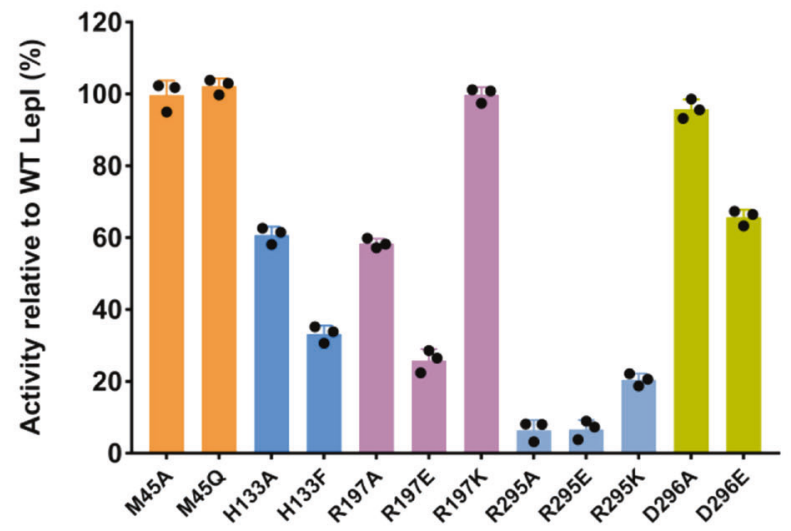

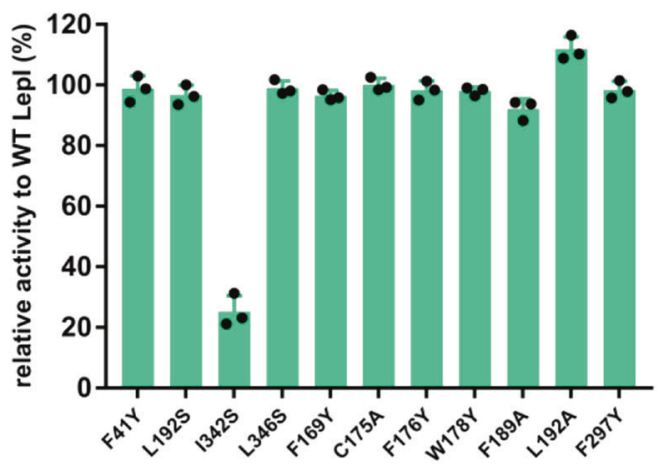

Fig. 4 Lepl catalyzes one step of the pericyclic reactions. a Overall structure of Lepl in complex with SAM and the precursor of Leporin C compound 1. Density maps are presented for SAM and precursor 1, which are colored in pink and blue, respectively. b The $2 F o-F C$ omit map, contoured at $1 \sigma$. The compound 1 molecule is shown in stick representation. c Close-up view of the substrate-binding site. The compound was coordinated at the substrate site, where four polar residues for substrate binding and catalysis surrounded by hydrophobic bulky residues with a semiopen configuration (Supplemental Fig. 8). d Enzymatic assays of the mutants around the substrate-binding site. H133A, R197A, and R295A greatly impaired the enzymatic activity. e Mutation of lle342 to serine greatly impaired the enzymatic activity, but most hydrophobic residues had little effect on the enzymatic activity

water, from entering randomly, thereby maintaining a hydrophobic habitat. To further investigate the role of positively charged residues (Arg197 and Arg295), we performed the DFT calculation by using ${ }^{+} \mathrm{NH}_{2}=\mathrm{CH}_{2}$ as a simpler mimic of arginine side chain. The barrier (TS-1) of the retro-Claisen rearrangement of 1 to the final HDA product 2 was substantially lowered by $4.9 \mathrm{kcal}$ $\mathrm{mol}^{-1}$, which proved the catalytic role of the positively charged residues around the substrate (Fig. 5a, b, Extended Data Table. 2). Thus, we propose that Lepl may facilitate retro-Claisen rearrangement through a combination of the following processes: (i) elimination of water molecules surrounding the substrate; (ii) stabilization of the reactive geometry, perhaps by decreasing the energy of retro-Claisen rearrangement via conformation immobilization; and (iii) enhancement of the reactivity by cationic residues.

\section{DISCUSSION}

The biochemical and structural analyses of the present study revealed that the functional evolution of a classic methyltransferase Lepl in fungi and the catalytic role of Lepl in retro-Claisen rearrangment reaction. The structure of SAM-bound Lepl suggests the dimeric or oligomeric state is essential for enzymatic activity, as indicated via an enzymatic assay involving $\mathrm{N}$-terminal deletion (Fig. 2d, e). SAM activates Lepl, whereas MTA and SAH decrease Lepl activity; however, SAH is a more-effective inhibitor than MTA, and MTA and SAH have synergistic effects on Lepl inhibition when simultaneously present (Fig. $1 \mathrm{~b}-\mathrm{d}$ ). These results imply that these inhibitions proceed through distinct mechanisms. Analyses of the Lepl crystal structures in complex with different compounds revealed that SAM stabilizes the Lepl structure and collaboratively participates the catalytic reaction as an activator, whereas SAH could partially inhibit the Lepl activity as an inhibitor, probably owing to the leakage of the sulfonium cation, whereas MTA, occupied the substrate site, as a competitive inhibitor (Figs. 1b-d and $2 \mathrm{~b}$ ). These findings were supported by the biochemical analyses (Fig. 1b-d). The structural conformational changes of these structures (Fig. 3d, e) indicate that several residues with different confirmations are present in the structures (Supplemental Fig. 6d). Additional analysis of trypsin resistance showed that Lepl stability may be further enhanced via MTA mimicry (Fig. 2f).

Positively charged residues interact with compound $\mathbf{1}$ and accelerate retro-Claisen rearrangement of IMDA adduct 1 to the final HAD product 2 (Supplemental Fig. 1), and the reaction is potentially accelerated 100-1000-fold by Lepl, and SAH can decelerate Lepl activity. However, our analyses suggest that the pericyclic reaction depends not only on the SAM but also on the presence of polar residues (His133, Arg197, Arg295, and Asp296) surrounding the substrate site, which allow substrate 1 to form preferentially in a lower-energy TS-1 configuration (Fig. 5a, b). Notably, analysis of enzyme kinetics revealed that SAM and SAH 


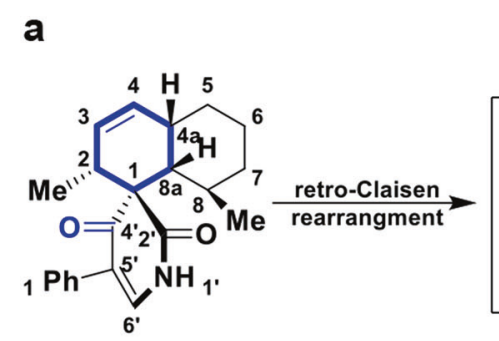

1

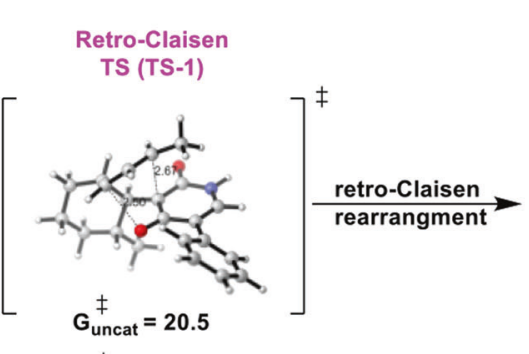

$G_{\text {cat }}^{\ddagger}=15.6$<smiles>C/C=C/[C@H]1Oc2c(-c3ccccc3)c[nH]c(=O)c2[C@H]2C[C@H](C)CCC[C@H]12</smiles>

2

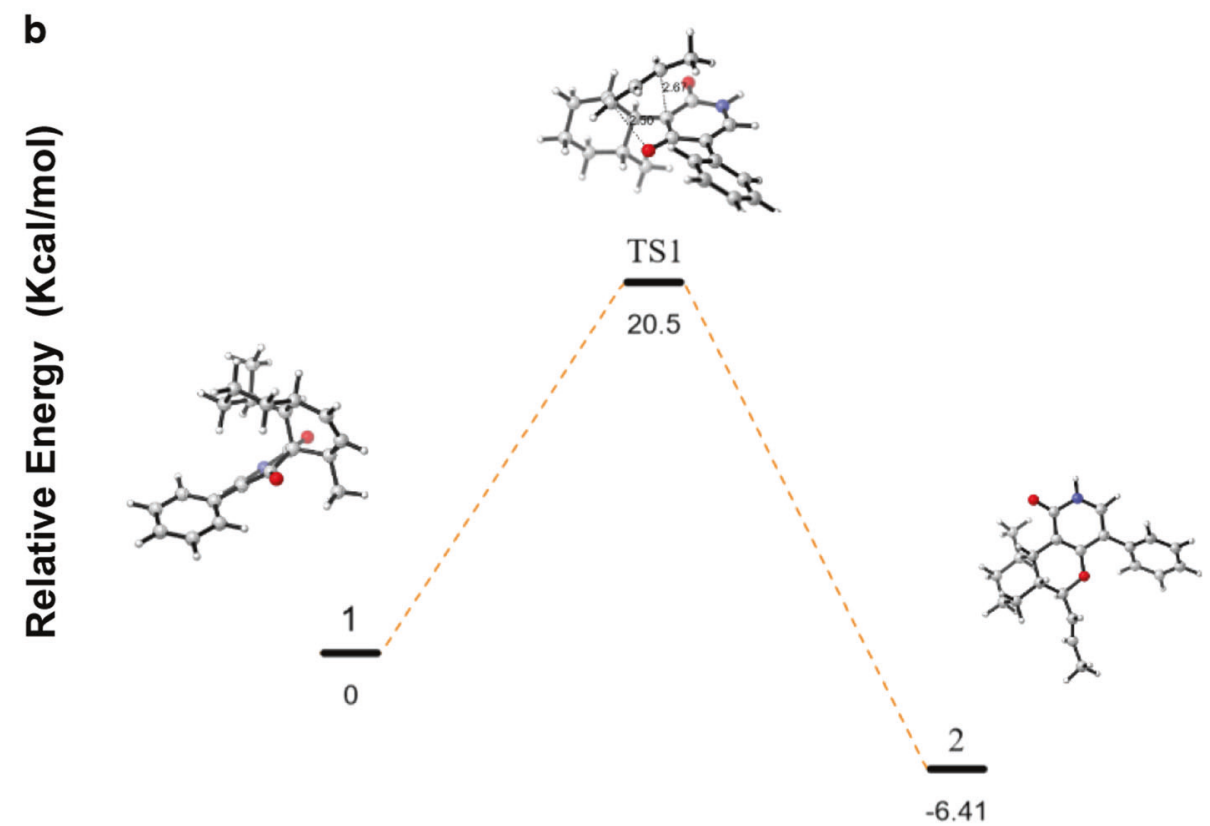

Fig. 5 DFT-computed free energies for the retro-Claisen rearrangement reactions. a Summary of Lepl-catalyzed reaction cascade leading from $\mathbf{1}$ to 2. b Free-energy diagram are shown for the non-enzymatic formation of $\mathbf{2}$ from $\mathbf{1}$. calculated with B3LYP-D3/6-31 G(d), gas phase. Numbers on levels show Gibbs free energies in $\mathrm{kcal} \mathrm{mol}^{-1}$

potentially alter the $K_{\mathrm{m}}$ of compound $\mathbf{1}$, and several critical residues also largely influence $K_{\mathrm{m}}$ values (Supplemental Fig. 9), consistent with structural observations.

Together, the present structural and biochemical analyses elucidate the possible mechanism underlying Lepl activation and extend our understanding of this enzyme, revealing a distinct regulatory mechanism. Similar SAM-dependent enzymes, most probably methyltransferases, have been studied extensively for decades, suggesting that the complexity of enzymatic mechanisms regulating activation and inhibition may have coevolved with fungi.

The present study reveals the novel catalytic mechanism underlying the pericyclic reaction of this enzyme. However, owing to limitations associated with intermediate structures, further studies are required to elucidate the detailed mechanisms underlying each catalytic step. Overall, our findings deepen our understanding of Lepl enzymatic activity and provide a fundamental basis for the rational design of natural-product derivatives for use in drug design and development.

\section{METHODS}

Chemicals

Compounds $\mathbf{1}$ and 3-5 were chemically synthesized according to the literature with minor modifications. ${ }^{32}$ NMR and HRMS spectroscopic data of Compound $\mathbf{2}$ was consistent with the report in the literature (Supplemental Fig. 10). ${ }^{22}$ Compound 1 was analyzed by MS spectroscopy and biochemical assay (Supplemental Fig. 11). HPLC-grade acetonitrile was purchased from Merck; water was purified and deionized by a water purification system from Biopak Milli-Q. All of the other chemicals used in this study were obtained from standard sources for laboratory use.

Truncation constructs and mutations

The N-terminal deleted truncations, including Lepl- $\Delta 15$ and Lepl$\Delta 37$, were designed based on the structure for disrupting the dimerization. The other mutations were performed for studying enzymatic activity. The primers for the mutations and truncations were all generated on the QuickChange Primer Design website, and the constructed plasmids were verified by Sanger sequencing.

Gene cloning, protein expression, and purification

A codon-optimized gene encoding Lepl, originated from A. flavus, was integrated into the expression vector pET-15b (Novagen, Madison, WI, USA) between the Ndel and Xhol sites with an $\mathrm{N}$ terminal histidine tag. DNA sequencing and transformation into Escherichia coli BL21 (DE3) cells for expression verified the constructed plasmid pET-15b-Lepl. A $100 \mathrm{ml}$ overnight culture was used to inoculate $6 \mathrm{~L}$ LB liquid medium supplemented with the essential antibiotic Ampicillin. When the cell density reached an OD $600 \mathrm{~nm}$ of 0.6 , the culture temperature was decreased from $37^{\circ} \mathrm{C}$ to $15^{\circ} \mathrm{C}$, and the culture was supplemented with $0.3 \mathrm{mmol} / \mathrm{L}$ isopropyl-d-1-thiogalactopyranoside to induce the expression of Lepl for $18 \mathrm{~h}$. Cell pellets harvested by $4000 \mathrm{rpm}$ centrifugation for 
$15 \mathrm{~min}$ were resuspended in $300 \mathrm{~mL}$ lysis buffer $(25 \mathrm{mmol} / \mathrm{L}$ Tris$\mathrm{HCl} \mathrm{pH} \mathrm{7.5,} 150 \mathrm{mmol} / \mathrm{L} \mathrm{NaCl}, 1 \mathrm{mmol} / \mathrm{L}$ phenylmethylsulfonyl fluoride) and disrupted using a low-temperature high-pressure cell disruptor. The lysate was clarified by centrifugation at $15,000 \mathrm{~g}$ for $45 \mathrm{~min}$ at $4{ }^{\circ} \mathrm{C}$, and the supernatant was loaded onto a column containing $5 \mathrm{~mL} \mathrm{Ni}$ resin. After an extensive wash with lysis buffer, the target protein Lepl was eluted with elution buffer $(25 \mathrm{mmol} / \mathrm{L}$ Tris- $\mathrm{HCl} \mathrm{pH} 7.5,150 \mathrm{mmol} / \mathrm{L} \mathrm{NaCl}, 250 \mathrm{mmol} / \mathrm{L}$ imidazole). The elution was concentrated to $2 \mathrm{~mL}$ and was loaded directly onto a HiLoad Superdex 200 10/300 GL size-exclusion chromatography column (GE Healthcare) in sample buffer $(10 \mathrm{mmol} / \mathrm{L}$ Tris- $\mathrm{HCl} \mathrm{pH}$ 7.5, $100 \mathrm{mmol} / \mathrm{L} \mathrm{NaCl}, 5 \mathrm{mmol} / \mathrm{L}$ dithiothreitol; DTT). The major peak fractions were analyzed with $12 \%$ sodium dodecyl sulfate polyacrylamide gel electrophoresis (SDS-PAGE). Selenomethionine Lepl was expressed in M9 medium supplemented with SeMet (Generon) to a final concentration of $100 \mu \mathrm{g} / \mathrm{mL}$ and purified similarly to the native proteins.

Crystallization, data collection, and structure determination

Purified Lepl was concentrated to $\sim 10 \mathrm{mg} / \mathrm{mL}$ in sample buffer $(10 \mathrm{mmol} / \mathrm{L}$ Tris- $\mathrm{HCl} \mathrm{pH} 7.5,100 \mathrm{mmol} / \mathrm{L} \mathrm{NaCl}, 5 \mathrm{mmol} / \mathrm{L}$ DTT). Crystallization conditions were initially determined with the sparse matrix screen (Hampton Research). Screening trials were established in 96 -well sitting-drop plates by combining $1 \mu \mathrm{L}$ of protein solution with an equal volume of well buffer. Crystals of Lepl were grown in buffer containing $0.15 \mathrm{~m}$ bis-Tris ( $\mathrm{pH} 7.0)$ and 40\% PEG 400. The crystals grew to their maximum size within 7 days. The crystals were mounted in a nylon loop and directly flash-frozen in liquid nitrogen. The diffraction data were collected at the Shanghai Synchrotron Radiation Facility at beamline BL19U1 and BL17U1 using a CCD detector. The diffraction images of Lepl were indexed, integrated and merged in XDS and scaled with SCALA as implemented in the xia2 package. ${ }^{33-35}$ Single-wavelength anomalous dispersion data were collected on a selenomethioninesubstituted Lepl crystal at the selenium absorption peak $(0.97918 \AA)$. The diffraction data were processed and scaled using HKL3000. ${ }^{36}$ The final model was manually constructed in $\mathrm{COOT}^{37}$ and refined in Phenix2. ${ }^{38}$ The SAH-Lepl and compound 1-bound Lepl structures were solved with molecular replacement (Molrep, Phenix2) with the corresponding SAM/MTA-Lepl structure, built in COOT, and refined by Phenix2. Data collection and processing statistics are summarized in online Table S1.

Activity assays of Lepl and its mutants

Assays for Lepl activity with mixture containing compound $\mathbf{1}$ in phosphate buffer $\left(20 \mathrm{~mm} \mathrm{Na}_{2} \mathrm{HPO}_{4}, 50 \mathrm{~mm} \mathrm{NaCl}, \mathrm{pH}\right.$ 8.0) were performed at the $50 \mu \mathrm{L}$ scale with 0.3 or $3 \mu \mathrm{m}$ Lepl at $30^{\circ} \mathrm{C}$ for $10 \mathrm{~min}$. Then, the reaction was quenched with a 3-equal volume of cold acetonitrile. Protein was precipitated and removed by centrifugation, and the supernatant was analyzed with an highperformance liquid chromatoggraphy (HPLC) system (Dionex UltiMate 3000, Thermo Scientific, USA) equipped with a LPG3400SDN pump system, a WPS-3000SL auto sampler with a $20 \mu \mathrm{L}$ injection loop, a TCC-3000RS column oven, and a VWD-3100 variable wavelength detector. A C18 column (Phenomenex Gemini-C18 column $150 \mathrm{~mm} \times 4.6 \mathrm{~mm}, 5 \mu \mathrm{m}$ ) was used with isocratic conditions ( $40 \%$ of $\mathrm{H}_{2} \mathrm{O}$ in $\mathrm{CH}_{3} \mathrm{CN}$ ). UV detection was performed at $260 \mathrm{~nm}$. Final results were calculated as percentage of control values. The error bars in the figures represent the standard deviation (S.D.) of three independent replicates. Data fitting was performed using GraphPad Prism 6.

Evaluation of SAH/MTA effects on retro-Claisen rearrangement Various concentrations of SAH (0-1 mM) or MTA (0-1 mM) were added to $50 \mu \mathrm{L}$ solutions containing Lepl in phosphate buffer (20 mM Na $2 \mathrm{HPO}_{4}, 50 \mathrm{~mm} \mathrm{NaCl}, \mathrm{pH} 8.0$ ) in the presence or absence of cofactors and incubated at RT for $10 \mathrm{~min}$. Then, the mixture containing compound $\mathbf{1}$ was added to the reaction mixture to initiate the enzymatic reaction. After $10 \mathrm{~min}$ of incubation at $30^{\circ} \mathrm{C}$, reactions were quenched by the adding $3 \times$ volume of acetonitrile. Protein was precipitated and removed by centrifugation, and the supernatant was analyzed by HPLC using a reversed-phase column ((Phenomenex Gemini-C18 column $150 \mathrm{~mm} \times 4.6 \mathrm{~mm}$, $5 \mu \mathrm{m})$ with isocratic conditions $\left(40 \%\right.$ of $\mathrm{H}_{2} \mathrm{O}$ in $\left.\mathrm{CH}_{3} \mathrm{CN}\right)$. Results were compared to the activity of WT-Lepl. Final results were calculated as percentage of control values. The error bars in the figures represent the standard deviation (S.D.) of three independent replicates. Data fitting was performed using GraphPad Prism 6.

Analytic size-exclusion chromatography

Proteins used in the experiments, including Lepl, Lepl-T3A/L10A/ Q13A/L14A, Lepl- $\Delta 15$, and Lepl- $\Delta 37-G S T$, were purified as described above. Pure Lepls were all adjusted to a final concentration of $1 \mathrm{mg} / \mathrm{mL}$ before injection into an analytical gelfiltration column (Superdex 200 Increase 10/30 GL; GE Healthcare) equilibrated with buffer containing $20 \mathrm{~mm} \mathrm{Na}{ }_{2} \mathrm{HPO}_{4}$ and $50 \mathrm{~mm} \mathrm{NaCl}(\mathrm{pH} 8.0)$. The migration positions of the Lepl variants were compared with the position of native-Lepl. The standard shift was monitored by injecting the following known proteins: thyroglobulin (bovine) $(670 \mathrm{kD}), \mathrm{\gamma}$-globulin (bovine) (158 kD), ovalbumin (chicken) (44 kD), myoglobin (horse) (17 kD), and vitamin B12 (1.35 kD).

Trypsin digestion assay

MTA, SAH, and SAM in $10 \mathrm{~mm}$ stock solution was added slowly to Lepl to achieve a final concentration of $1 \mathrm{~mm}$, and the mixture was incubated on ice for $10 \mathrm{~min}$. Trypsin stock solutions were prepared in Tris-Mg solution $(10 \mathrm{mg} / \mathrm{mL})$ and twofold serially diluted to eight different concentrations in buffer containing $20 \mathrm{~mm}$ $\mathrm{Na}_{2} \mathrm{HPO}_{4}$ and $50 \mathrm{~mm} \mathrm{NaCl}(\mathrm{pH}$ 8.0). Then, the trypsin was added to a final protease-to-protein ratio of $1: 5(\mathrm{v} / \mathrm{v})$ and incubated on ice for $30 \mathrm{~min}$. Loading buffer was added to the mixture to a final protease-to-ratio of $1: 1(\mathrm{v} / \mathrm{v})$ at $100^{\circ} \mathrm{C}$ for $10 \mathrm{~min}$, and the mixture was then analyzed via $15 \%$ SDS-PAGE.

Sequence alignment

Multiple sequence alignments were generated on the ClustalW online service and edited using the ESPript 3.0 program. ${ }^{39}$

\section{DATA AVAILABILITY}

The coordinates of the structures have been deposited in the Protein Data Bank (PDB) under accession codes 6J10 for MTA/SAM-Lepl, 6J24 for SAM/ligand 1-bound Lepl, and $6 \mathrm{~J} 46$ for SAH-Lepl. Other data that support the findings of this study are available from the corresponding author upon request.

\section{ACKNOWLEDGEMENTS}

We thank the staff of the BL17U1 beamline and BL19U1 at the Shanghai Synchrotron Radiation Facility, Zhangjiang Lab, for assistance during data collection. We gratefully acknowledge the financial support from the National Natural Science Foundation of China (Grant No. 31570842, No. 31870836, and No. 21702141), and the National Young Thousand Talents Program and the Sichuan Province Thousand Talents program in China.

\section{AUTHOR CONTRIBUTIONS}

W.C. and X.W. designed the research; Q.S. and Y.H. made the constructs, purified the proteins, grew and optimized the crystals, Q.S., Y.Y., and S.Y. performed activity assays.; J.H. and Q.S. synthesized the compounds; J.H., L.L, and T.W. made computational calculation. Q.S., Y.H., C.D.; Y.G. collected the data; Y.G. and C.W. determined the structure; X.F., L.H., S.Q., X.Z., S.Y., and Y.W. contributed materials and data analysis. W.C. wrote manuscript with contributions from the other authors. 


\section{ADDITIONAL INFORMATION}

The online version of this article (https://doi.org/10.1038/s41392-019-0052-y) contains supplementary material, which is available to authorized users.

Competing interests: The authors declare no competing interests.

\section{REFERENCES}

1. Erb, A., Sturmer, T., Marre, R. \& Brenner, H. Prevalence of antibiotic resistance in Escherichia coli: overview of geographical, temporal, and methodological variations. Eur. J. Clin. Microbiol. Infect. Dis. 26, 83-90 (2007).

2. Durdu, B. et al. Risk factors affecting patterns of antibiotic resistance and treatment efficacy in extreme drug resistance in intensive care unit-acquired klebsiella pneumoniae infections: a 5-year analysis. Med. Sci. Monit. 25, 174-183 (2019).

3. Kuehn, B. Antibiotic resistance challenge. JAMA 320, 1851 (2018).

4. Bao, J. et al. Bioactive pyridone alkaloids from a deep-sea-derived fungus arthrinium sp. UJNMF0008. Mar. Drugs 16, pii: E174 (2018).

5. Fujita, Y., Oguri, H. \& Oikawa, H. Biosynthetic studies on the antibiotics PF1140: a novel pathway for a 2-pyridone framework. Tetrahedron Lett. 46, 5885-5888 (2005).

6. Wang, Y. T., Xue, Y. R. \& Liu, C. H. A brief review of bioactive metabolites derived from deep-sea fungi. Mar. Drugs 13, 4594-4616 (2015).

7. Bills, G. F. et al. Enhancement of antibiotic and secondary metabolite detection from filamentous fungi by growth on nutritional arrays. J. Appl. Microbiol. 104, 1644-1658 (2008).

8. Wang, J. F. et al. Arthpyrones A-C, pyridone alkaloids from a sponge-derived fungus arthrinium arundinis ZSDS1-F3. Organic Lett. 17, 656-659 (2015).

9. Li, L. N. et al. Discovery and characterization of 4-hydroxy-2-pyridone derivative sambutoxin as a potent and promising anticancer drug candidate: activity and molecular mechanism. Mol. Pharm. 15, 4898-4911 (2018).

10. Bihani, M. \& Zhao, J. C. G. Advances in asymmetric diastereodivergent catalysis. Adv. Synth. Catal. 359, 534-575 (2017).

11. Xiao, W. et al. Regio- and diastereodivergent [4+2] cycloadditions with cyclic 2,4-dienones. Org. Lett. 20, 236-239 (2018).

12. Fernandez-Garcia, J. M., Fernandez-Rodriguez, M. A. \& Aguilar, E. Catalytic intermolecular hetero-dehydro-diels-alder cycloadditions: regio- and diasteroselective synthesis of 5,6-dihydropyridin-2-ones. Organic Lett. 13, 5172-5175 (2011).

13. Diesel, J., Finogenova, A. M., Cramer, N. \& Nickel-Catalyzed Enantioselective Pyridone, C.-H. Functionalizations enabled by a bulky N-heterocyclic carbene ligand. J. Am. Chem. Soc. 140, 4489-4493 (2018).

14. Jessen, H. J. \& Gademann, K. 4-Hydroxy-2-pyridone alkaloids: structures and synthetic approaches. Nat. Prod. Rep. 27, 1168-1185 (2010).

15. Lin, C. I., McCarty, R. M. \& Liu, H. W. The enzymology of organic transformations: a survey of name reactions in biological systems. Angew. Chem. Int. Ed. Engl. 56, 3446-3489 (2017).

16. Stocking, E. M. \& Williams, R. M. Chemistry and biology of biosynthetic Diels-Alder reactions. Angew. Chem. Int. Ed. Engl. 42, 3078-3115 (2003).

17. Oikawa, H. \& Tokiwano, T. Enzymatic catalysis of the Diels-Alder reaction in the biosynthesis of natural products. Nat. Prod. Rep. 21, 321-352 (2004).

18. Kim, H. J., Ruszczycky, M. W., Choi, S. H., Liu, Y. N. \& Liu, H. W. Enzyme-catalysed $[4+2]$ cycloaddition is a key step in the biosynthesis of spinosyn A. Nature $\mathbf{4 7 3}$, 109-112 (2011).

19. Byrne, M. J. et al. The catalytic mechanism of a natural diels-alderase revealed in molecular detail. J. Am. Chem. Soc. 138, 6095-6098 (2016).

20. Minami, A. \& Oikawa, H. Recent advances of Diels-Alderases involved in natural product biosynthesis. J. Antibiot. (Tokyo) 69, 500-506 (2016).
21. Medvedev, M. G. et al. Quantifying possible routes for SpnF-catalyzed formal diels-alder cycloaddition. J. Am. Chem. Soc. 139, 3942-3945 (2017)

22. Ohashi, M. et al. SAM-dependent enzyme-catalysed pericyclic reactions in natural product biosynthesis. Nature 549, 502-506 (2017).

23. Cary, J. W. et al. An Aspergillus flavus secondary metabolic gene cluster containing a hybrid PKS-NRPS is necessary for synthesis of the 2-pyridones, leporins. Fungal Genet. Biol. 81, 88-97 (2015).

24. Glockle, A. \& Gulder, T. A. M. A pericyclic reaction cascade in leporin biosynthesis. Angew. Chem. Int. Ed. Engl. 57, 2754-2756 (2018).

25. Ruszczycky, M. W. \& Liu, H. W. Mechanistic enzymology of the radical SAM enzyme Desll. Isr. J. Chem. 55, 315-324 (2015).

26. Broderick, W. E., Hoffman, B. M. \& Broderick, J. B. Mechanism of radical initiation in the radical S-adenosyl-I-methionine superfamily. Acc. Chem. Res. 51, 2611-2619 (2018).

27. Coward, J. K. \& Slisz, E. P. Analogs of S-adenosylhomocysteine as potential inhibitors of biological transmethylation. Specificity of the S-adenosylhomocysteine binding site. J. Med. Chem. 16, 460-463 (1973).

28. Vidgren, J., Svensson, L. A. \& Liljas, A. Crystal structure of catechol Omethyltransferase. Nature 368, 354-358 (1994).

29. Jiang, J. et al. Functional and structural analysis of phenazine O-methyltransferase LaPhzM from Lysobacter antibioticus $\mathrm{OH} 13$ and one-pot enzymatic synthesis of the antibiotic Myxin. ACS Chem. Biol. 13, 1003-1012 (2018).

30. Singh, S. et al. Structural characterization of the mitomycin 7-Omethyltransferase. Proteins 79, 2181-2188 (2011).

31. Newmister, S. A. et al. Unveiling sequential late-stage methyltransferase reactions in the meleagrin/oxaline biosynthetic pathway. Org. Biomol. Chem. 16, 6450-6459 (2018).

32. Snider, B. B. \& Lu, Q. Total synthesis of (+/-)-leporin A. J. Org. Chem. 61, 2839-2844 (1996).

33. Winter, G., Lobley, C. M. \& Prince, S. M. Decision making in xia2. Acta. Crystallogr. D Biol. Crystallogr. 69, 1260-1273 (2013).

34. Evans, P. Scaling and assessment of data quality. Acta. Crystallogr. D Biol. Crystallogr. 62, 72-82 (2006).

35. Kabsch, W. XDS. Acta Crystallogr. D Biol. Crystallogr. 66, 125-132 (2010).

36. Otwinowski, Z. \& Minor, W. Processing of X-ray diffraction data collected in oscillation mode. Methods Enzymol. 276, 307-326 (1997).

37. Emsley, P., Lohkamp, B., Scott, W. G. \& Cowtan, K. Features and development of Coot. Acta Crystallogr. D Biol. Crystallogr. 66, 486-501 (2010).

38. Afonine, P. V. et al. Towards automated crystallographic structure refinement with phenix.refine. Acta Crystallogr. D Biol. Crystallogr. 68, 352-367 (2012).

39. Larkin, M. et al. Clustal W and Clustal X version 2.0. Bioinformatics 23, 2947-2948 (2007).

(i) Open Access This article is licensed under a Creative Commons adaptation, distribution and reproduction in any medium or format, as long as you give appropriate credit to the original author(s) and the source, provide a link to the Creative Commons license, and indicate if changes were made. The images or other third party material in this article are included in the article's Creative Commons license, unless indicated otherwise in a credit line to the material. If material is not included in the article's Creative Commons license and your intended use is not permitted by statutory regulation or exceeds the permitted use, you will need to obtain permission directly from the copyright holder. To view a copy of this license, visit http://creativecommons. org/licenses/by/4.0/.

(c) The Author(s) 2019 